\title{
COMPARATIVE ANALYSIS OF THE EFFECTIVENESS OF CHRONOTHERAPY AND TRADITIONAL METHOD OF ALOPECIA AREATA TREATMENT
}

\author{
Nikolay Polischuk \\ Chronomedicine Clinic «Rezonans» \\ 3 Geroiv Oborony str., Kyiv, Ukraine, 03150 \\ rezonansua@gmail.com \\ Ilya Polischuk \\ Chronomedicine Clinic «Rezonans» \\ 3 Geroiv Oborony str., Kyiv, Ukraine, 03150 \\ rezonansua@gmail.com
}

\begin{abstract}
Alopecia areata - is the one of widespread baldness forms, difficultly subjected to treatment. The method of chronotherapy that gains an essential circulation in Ukraine and throughout the world may play the important role in its solution.

Aim: to study the clinical effectiveness of chronotherapy for alopecia areata treatment.

Materials and methods. For attaining the set aim 108 persons with the diagnosis alopecia areata were examined and treated. Patients were divided in two groups. The main group included 45 persons - treated using the method of chronotherapy. The control group consisted of 67 persons, who received the traditional treatment of alopecia areata. The series of clinical, biochemical and immunological studies, directed on the assessment of the treatment effectiveness, were realized.

Results. The treatment of patients with alopecia areata by the method of chronotherapy is statistically reliably more effective than the traditional treatment of this pathology and provides the renewal of hair growth in 53,3 \% of patients that is accompanied by normalization of alexin, sulfhydryl groups content, acid phosphatase activity, serine, asparagine acid, valine, threonine, alanine, cystine, leucine, isoleucine concentrations. The offered chronotherapy method has the expressed anabolic effect on patients' organism. The menstrual cycle is normalized, pre-menstrual syndrome symptoms and aldodysmenorrhea disappear, patients' working ability and state of health improve.

Conclusions. The obtained data deepen knowledge about the pathogenesis of alopecia areata at the biochemical and immunological levels and favor the rise of the effectiveness of diagnostics and treatment of this pathology. At the study the high chronotherapy effectiveness at treating alopecia areata was revealed and the possibility of its use for the effective treatment of a series of other pathologies was proved.
\end{abstract}

Keywords: chronotherapy, traditional treatment method, alopecia areata, treatment effectiveness, immunological indices, biochemical indices.

\section{Introduction}

Alopecia areata - is the one of widespread forms of alopecia, characterized with a total loss of long hair at distinctly limited skin areas, of round or oval outlines, in average $3-5 \mathrm{~cm} \mathrm{di-}$ ameter. For this disease is typical the occasional start, most often in young persons of both sexes. Numerous facts indicate that this type of alopecia is a result of autoimmune, psycho-neurogenic, toxic factors. The appearance of alopecia areata can be also a symptomatic sign of some infectious diseases (engirdling herpes, syphilis and so on), inflammatory processes on skin (eczema, pyodermias) $[1,2]$. But causes of appearance and the development mechanism of alopecia areata are not completely elucidated and need the further study.

Despite the fact that alopecia is considered as a relatively benignant disease, hair loss leads to severe changes in the phychoemotional status, up to serious neurotic disorders and formation of a pathologic personality [3,4]. It conditions the essential social importance of this disease and necessity of its effective treatment.

Despite the wide use of synthetic drugs (minoxidil, finasteride, pepper-camphoric liniment, zincteral, etonium, vitamin complex "Perfectil" and so on) and active use of medical and cosmetic preparations on the base of medical herbs at alopecia treatment, it remains insufficiently effective. 
Chronotherapy can help to solve this problem at the expanse of raising the effectiveness of known therapeutic means and decreasing their side effects as a result of more adequate, physiologically grounded choice of doses and time of drugs intake.

Although chronotherapy is rather young science, the active work of researchers allows to make a series of interesting discoveries in this field for today.

Alongside with theoretical studies, chronotherapy has for today essential practical results. Surprisingly, preparations, acknowledged as ineffective ones, demonstrate good results at using chronotherapy. The method of chronotherapy is in fact that drugs must be taken at a proper time that is connected with a biorhythm of an organ that the treating effect is directed on.

This direction of medicine is now on the peak of its development in the USA, Germany, England and so on. In the last years essential progress is observed in therapy, dermatology, oncology, rheumatology, surgery. Chronotherapy has the great effect at treating different chronic diseases, just in cases, when traditional medical methods are not able to cope with a disease [5].

In Ukraine and in the whole world chronotherapeutic treatment schemes gain more circulation, and their effect is essentially higher comparing with one of the use of traditional treatment schemes [6].

Epidemiological and experimental studies testify to the role of circadian disorders in the development of many diseases, not only dermatological, but a series of other ones, especially oncologic [7]. Sigurdardottir LG and co-authors revealed the mutual dependence between the circadian rhythms failure, sleep disorder (lack of sleep) and prostate cancer at epidemiological studies [8]. Relogio and co-authors indicate the direct mutual dependence between the circadian rhythms imbalance and rectum cancer [9].

Individual chronotherapy allows to choose the scheme of drugs administration, individually for each patients, taking into account features of his/her day rhythms. Due to this fact, specialists achieved essential successes in treating a series of diseases, especially hypertonic disease and bronchial asthma.

Thus, Smolensky M. N. and co-authors in their studies received convincing data that testify to the decrease of the risk of cardiovascular diseases and lesions of organ-targets of blood vessels and heart tissue, brain, kidneys, eyes and other organs at using chronotherapeutic methods at arterial hypertension treatment [10]. The effectiveness of the use of chronotherapy principles in the treatment of hypertonic disease was demonstrated in researches by Hermida R. C. and co-authors by proving and substantiating that the intake of such antihypertensive preparations as amlodipine and valsartan before sleep gives more expressed effect than their intake mornings [11]. Correspondent data about the dependence of the preparation effectiveness on the time of its intake were obtained also for the combination of indapadide and valsartan [12] and for the combination of valsartan and hydrochloride [13].

In a series of researchers was revealed the presence of circadian rhythms at obstruction of respiratory tracts at pulmonary chronic obstructive diseases [14] and especially asthma [15]. It conditions the necessity of wide introduction of chronotherapy methods in COPD treatment schemes.

The important role is played by circadian rhythms also in the process of organism ageing. Thus, in a series of researches was found the direct interconnection between the circadian rhythms imbalance and human ageing speeds at the molecular level [16].

Taking into account the aforesaid, the research, directed on the study of methods of alopecia areata treatment methods, is urgent.

\section{Aim of research}

To compare the clinical effectiveness of chronotherapetic and traditional (conventional) methods of alopecia areta treatment.

\section{Materials and methods}

The study was carried out on the base of the department of dermatology and venereology of NMU, named after O. O. Bogomolets and clinic of chronomedicine "Resonance" (Kyiv city) in the period of 1986-2016 years. 
For attaining the set aim 108 persons with the diagnosis alopecia areata were examined and treated. Patients were divided in two groups. The main group included 45 persons - treated using the method of chronotherapy. The control group consisted of 67 persons, who received the traditional treatment of alopecia areata.

Patients from the main group received officinal tinctures of adaptogens as medical preparations in different doses by the individual scheme, elaborated according to the month cycle of hair growth. After finishing 30-days treatment course, the treatment scheme was repeated many times during 5-10 courses. Patients from the control group received the complex of therapeutic preparations that included methionine, zinc oxide, apilak, adaptogens tincture, injections of plasmol, aloe, vitamins, physiotherapeutic preparations. The treatment by this method was realized during 1 month.

All experimental persons underwent a series of biochemical and immunologic examinations before and after treatment, especially there was determined the level of general, albuminous and exalbuminous sulfhydryl groups, free amino acids concentrations (asparagine acid, serine, glutamine acid, proline, valine, threonine, glycine alanine, cystine, leucine, isoleucine, tyrosine, phenylalanine), activity of acid and alkaline phosphatases, level of alexin and alexin-binding antibodies to skin tissues in the liver, immunoglobulins of $\mathrm{A}$ and $\mathrm{M}$ classes, immunoglobulin-antibodies of main classes to the skin and liver, $\mathrm{C}$ cytochrome, activity of T- and B-lymphocytes.

The content of general and exalbuminous sulfhydryl groups was determined by the method, offered by L. L. Elman and G. Seldak. The activity of acid and alkaline phosphotases in blood serum was determined by Bogdansky's method. The determination of the concentration of free amino acids was realized by the method of ion-exchanging chromatography on automatic analyzers by the Japanese firm "Hitachi" KLA-3 and KLA-5. The determination of immunoglobulins of A and M classes was realized by the method of simple radial immunodiffusion by G. Manchini. For determining humoral antibodies in blood serum the reaction of immunoglobulins consumption was used.

The alexin content in blood serum and the speed of alexin use with skin and liver antibodies were determined by the method of V. L. Morozov. Blood sampling for immunological and biochemical tests was realized mornings. Blood was taken from the cubital vein on an empty stomach.

The treatment clinical effectiveness was assessed by the scale as perfectly, good or satisfactory. The perfect effect presupposed the full renewal of hair cover, the good one - essential decrease of the cosmetic defect that favored the renewal of the face look, typical for each person. The satisfactory effect was considered as eyelashes, eyebrows growth, partial hair renewal, that didn't essentially influence the patient's look normalization.

\section{Results}

At preliminary (at the stage of primary examination) biochemical studies it was revealed, that in blood serum of patients with alopecia areata was 2,4 times less methionine, by 2,5 ptimes glutamine acid, comparing with the norm; at the same time, by $89 \%$ less cystine, by $74 \%$ - alanine, by $50 \%$ - leucine and asparagine acid, by $40 \%$ less serine. In blood serum were by $40 \%$ more general sulfhydryl groups, comparing with the norm, including by $84,9 \%$ more albuminous ones and by $36 \%$ more exalbuminous ones. The activity of acid phosphotase exceeded their activity in healthy persons in 4,6 and 2,7 times respectively.

Alongside with it, in examined persons was revealed the decrease of alexin content and increase of alexin-binding antibodies to the liver tissue in 5 times, to skin tissues - in 14,4 times comparing with the norm. There was also revealed the increase of the level of immunoglobulin of A class by $50 \%$, and immunoglobulin of $\mathrm{M}$ class - by $27 \%$, abrupt increase of the content of anti-kidney immunoglobulin-A-antibodies and also the increase of immunoglobulin-A-antibodies to $\mathrm{C}$ cytochrome in 2,2 times.

The analysis of data of the assessment of the clinical effectiveness of chromatography and traditional method of alopecia areata treatment demonstrated that at chronotherapy the perfect result was detected in 24 patients of 45 , that is in 53,3\% of persons, good - in 7 patients, or 15,6; \% of treated persons, satisfactory - in 9 patients, or in $20 \%$ of persons, unsatisfactory - in 5 patients or in $11,1 \%$ of them. At the treatment by the conventional method the full hair renewal was detected in 3 patients of 67 (4,5\%), good effect was obtained in 9 patients $(13,4 \%)$, satisfactory - in 12 patients (17,9\%), unsatisfactory - in 43 patients (64,2\%). These data are presented visually on Fig. 1. 


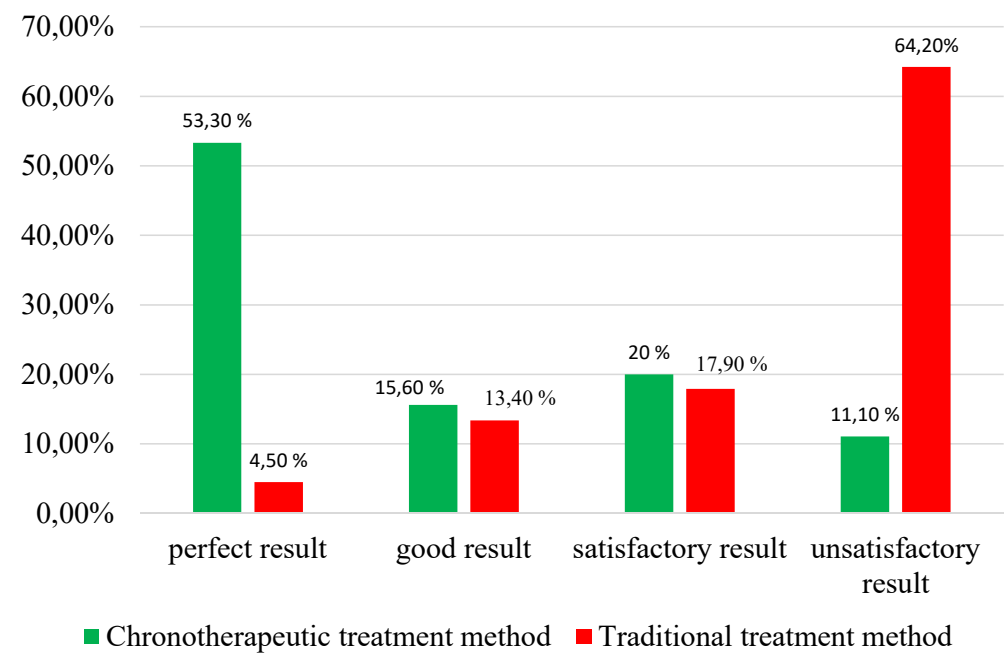

Fig. 1. Clinical effectiveness of chronotherapeutic and traditional treatment methods

Statistical calculations with the determination of Student t-criterion demonstrated that the positive treatment effect at chronotherapy 2,5 times exceeds the one at the conventional treatment method with the high reliability degree $(\mathrm{P}<0,001)$. At that the perfect result at chronotherapy was detected 11,8 times more often than at the conventional method. The good result was detected by $16,4 \%$ more often, satisfactory one - by $11,7 \%$ more often.

Alongside with the hair growth stimulation in patients with alopecia areata at chronotherapy were observed also other effects such as the menstrual cyclicity normalization, pre-menstrual syndrome and algodismenorrhea disappearance, sleep and appetite normalization, disappearance of headaches, vision improvement, apathy disappearance, working ability improvement and so on.

Alongside with it chronotheraphy, as opposite to the conventional method, favors the decrease of the content of general sulfhydryl groups in patients' blood serum to almost physiological level that is connected with the decrease of the quantity of albuminous and exalbuminous organic compounds. The activity of acid and alkaline photophases is also normalized. It testifies to the fact that chronotherapy has the more expressed ability to normalize metabolic processes in patients' organism. The analysis of the content of free amino acids in blood serum of patients, treated by different methods, demonstrated the tendency to decrease of the concentration of most amino acids in blood serum such as asparagine acid, serine, glutamine acid, valine, alanine, cystine, leucine, isoleucine during the first 7 months of chronotherapy treatment.

After the first 10 months of treatment the content of asparagine acid, serine, threonine, valine, alanine, cystine, leucine, isoleucine in blood serum is normalized, and the content of proline, tyrosine and phenylalanine even increased.

At the same time the conventional treatment method, despite the mass character of the therapeutic influence, favored the normalization of free amino acids in patients' organism much less.

Under influence of chronotherapy just in 1 month afer the start of treatment the content of alexin and $\mathrm{A}$ and $\mathrm{M}$ immunoglobulins in patients increases almost to the norm. In next 10 months the level of alexin and immunoglobulins continues to increase, whereas at treatment by the conventional method these indices are not normalized.

Under the influence of chronotherapy the level of anti-kidney and anti-liver immunoglobulin-A-antibodies decreased to the norm, in first months of observation less, at following ones more. The obtained data testify to the more expressed positive influence of chronotherapy on immunological processes that take place in the liver than at the conventional treatment method.

Under chronotherapy influence takes place the essential decrease of the concentration of immunoglobulin-A and $\mathrm{M}$-antibodies to $\mathrm{C}$ cytochrome, especially expressed at 4, 7 and 10-th months of treatment. Thus, the dynamics of most immunologic indices at treating patients with alopecia areata testify to their more expressed normalization at using chromatography comparing with the conventional treatment method. 
In whole the dynamics of results of biochemical and immunological studies proves that chronotherapy has the anabolic effect on the patents' organism and testify to the correctness of theoretical elaborations that were the base for the offered treatment method creation.

\section{Discussion of results}

The obtained research data about changes of biochemical and immunological indices in patients with alopecia areata essentially deepen knowledge about its pathogenesis and, as a result, open wide possibilities for the further search for the first cause (main etiological factor) of this disease development, elaboration of new methods of its diagnostics, new more effective, easy, available and safe treatment methods.

The one of such treatment methods it is the intake of adaptogens tinctures using chromatography methods - individual choice of doses and time of intake taking into account the hair growth cyclicity of a patient. The more effectiveness of this treatment method comparing with traditional treatment was proved in this research.

At the same time this treatment method demonstrated the expressed anabolic effect on patients' organism. Their menstrual cycle was normalized, pre-menstrual syndrome and algodismenorrhea symptoms disappeared, working ability and health status improved.

The data, accordant to the results of this study were found in scientific literature. Thus, Garanyan L. G. presents the data about the high effectiveness of chronotherapeutic methods using melatonin in the treatment of a series of dermatologic diseases: psoriasis, eczema, atopic dermatitis and, especially alopecia [17].

A lot of attention in scientific literature is also paid to the problematic of the study of chronobiology of the human immune system to use obtained data for creating effective diagnostic and treatment chronotherapeutic methods. Thus, Borodin Y. I. writes that at disorders of circadian rhythms of the immune system in experiments on animals and at different human diseases, disorders of immunocompetent cells functions, manifested by immunodeficiency, autoimmune, allergic states in patients, are observed [18]. Already today the important role in the treatment of diseases of autoimmune character, especially rheumatoid arthritis, is given to chronotherapy [19].

The extremely important role of circadian rhythms in the development of dermatological diseases and importance of chronotherapy approaches at this problem solution are considered also in the article by Li WQ and co-authors [20].

Based on the aforesaid, we can talk about perspectiveness of chronobiology, chronomedicine, chronodiagnostics and chronotherapy and their important role in the further development of the modern medicine. It is extremely important to realize the great number of researches, directed on the search for new methods of diagnostics and treatment of diseases, especially, dermatologic ones, based on chronomedicine principles.

The research, presented in this study, is one of them. The further study of this problematic will allow to widen our knowledge about alopecia and will help to find the most effective, most simple and most available method of its treatment, and also to widen our knowledge of chronomedicine that the future of the modern medicine is connected with. The researches, directed on the study of the effectiveness of other methods of alopecia treatment, especially traditional ones, with their accordance to the principles of chronomedicine, seem to be perspective.

\section{Conclusions}

1. The obtained research data about pathological changes of biochemical and immunological indices in patients with alopecia areata deepen knowledge about the pathogenesis of alopecia areata and favor the rise of the effectiveness of diagnostics and treatment of this pathology.

2. The treatment of patients with alopecia areata by the method of chronotherapy is more effective than the traditional treatment of this pathology and provides the full renewal of the hair cover in $53,3 \%$ of patients that is accompanied by normalization of the content of alexin, sulfhydryl groups, acid phosphotase activity, concentrations of serine, asparagine acid, valine, threonine, alanine, cystine, leucine, isoleucine. 
3. The offered chronotherapy treatment method has the expressed anabolic effect on patients' organism. Their menstrual cycle was normalized, pre-menstrual syndrome and algodismenorrhea symptoms disappeared, working ability and health status improved.

4. The high therapeutic effectiveness, good reaction and availability of methods of chronotherapy of alopecia areata that are in the individual choice of doses and time of drugs intake by patients depending on their biological rhythms, especially, cyclicity of hair growth allow to recommend them for the wide use under both stationary and outpatient conditions.

\section{References}

[1] Soloshenko, E. N. (2009). Clinical varieties of alopecia: pathogenesis, differential diagnosis, therapy. International Medical Journal, 1, 102-109.

[2] Wang, E., Mcelwee, K. J. (2011). Etiopathogenesis of alopecia areata: Why do our patients get it? Dermatologic Therapy, 24 (3), 337-347. doi: 10.1111/j.1529-8019.2011.01416.x

[3] Olisova, O. Yu., Verkhozhdory, I. V., Gostroverkhova, I. P. (2010). Contemporary notions about etiology, pathogenesis and treatment of focal alopecia. Journ Skin and venereal diseases, 1, 48-52.

[4] Serbina, I. M. (2014). Cytokine-mediated mechanisms of formation of alopecia areata. Scientific bulletins of BelSU. Series: Medicine. Pharmacia, 28 (24 (195)), 32-38.

[5] Boger, Ts. M. (2000). Duration of drugs and moon phases. Krivoy Rog: Rimedia Gomeo, 108.

[6] Degtyareva, Z. Ya., Moiseev, N. I., Fedoseev, G. B. (1981). Chronobiological approach in the clinic and therapy of bronchial asthma. Chronobiology and Chronopathology. Moscow, 923.

[7] Smolensky, M. H., Hermida, R. C., Ayala, D. E., Tiseo, R., Portaluppi, F. (2010). Administration-time-dependent effects of blood pressure-lowering medications: basis for the chronotherapy of hypertension. Blood Pressure Monitoring, 15 (4), 173-180. doi: 10.1097/mbp.0b013e32833c7308

[8] Hermida, R. C., Ayala, D. E., Calvo, C., Portaluppi, F., Smolensky, M. H. (2007). Chronotherapy of hypertension: Administration-time-dependent effects of treatment on the circadian pattern of blood pressure. Advanced Drug Delivery Reviews, 59 (9-10), 923-939. doi: 10.1016/j.addr.2006.09.021

[9] Huangfu, W., Duan, P., Xiang, D., Gao, R. (2015). Administration time-dependent effects of combination therapy on ambulatory blood pressure in hypertensive subjects. International Journal of Clinical and Experimental Medicine, 8 (10), 19156-19161.

[10] Hermida, R. C., Ayala, D. E., Mojon, A., Fontao, M. J., Fernandez, J. R. (2011). Chronotherapy With Valsartan/Hydrochlorothiazide Combination in Essential Hypertension: Improved Sleep-Time Blood Pressure Control With Bedtime Dosing. Chronobiology International, 28 (7), 601-610. doi: 10.3109/074205 28.2011.589935

[11] Ciappi, G., De Benedetto, F., D’Illario, F. et. al. (1982). Chronobiological aspects of bronchial tone. Chronobiologia, 9 (2), 163-172.

[12] Hetzel, M. R., Clark, T. J. (1980). Comparison of normal and asthmatic circadian rhythms in peak expiratory flow rate. Thorax, 35 (10), 732-738. doi: 10.1136/thx.35.10.732

[13] Sigurdardottir, L. G., Valdimarsdottir, U. A., Fall, K., Rider, J. R., Lockley, S. W., Schernhammer, E., Mucci, L. A. (2012). Circadian Disruption, Sleep Loss, and Prostate Cancer Risk: A Systematic Review of Epidemiologic Studies. Cancer Epidemiology Biomarkers \& Prevention, 21 (7), 1002-1011. doi: 10.1158/1055-9965.epi-12-0116

[14] Lengyel, Z., Battyani, Z., Szekeres, G., Csernus, V., Nagy, A. D. (2013). Circadian clocks and tumor biology: What is to learn from human skin biopsies? General and Comparative Endocrinology, 188, 67-74. doi: 10.1016/j.ygcen.2013.03.033

[15] Relogio, A., Thomas, P., Medina-Perez, P., Reischl, S., Bervoets, S., Gloc, E. et. al. (2014). Ras-Mediated Deregulation of the Circadian Clock in Cancer. PLoS Genetics, 10 (5), e1004338. doi: 10.1371/ journal.pgen.1004338

[16] Khapre, R. V., Samsa, W. E., Kondratov, R. V. (2010). Circadian regulation of cell cycle: Molecular connections between aging and the circadian clock. Annals of Medicine, 42 (6), 404-415. doi: 10.3109/ 07853890.2010 .499134

[17] Garanyan, L. G. (2015). Melatonin preparations in dermatological practice. Russian journal of skin and venereal diseases, $18(6), 50-52$. 
[18] Borodin, Yu. I., Trufakin, V. A., Michurina, S. V., Shurlygina, A. V. (2012). Structural and temporal organization of liver, lymphatic, immune and endocrine systems in violation of the light regime and the administration of melatonin. Novosibirsk: Manuskript, 207.

[19] Cutolo, M. (2012). Chronobiology and the treatment of rheumatoid arthritis. Current Opinion in Rheumatology, 24 (3), 312-318. doi: 10.1097/bor.0b013e3283521c78

[20] Li, W.-Q., Qureshi, A. A., Schernhammer, E. S., Han, J. (2013). Rotating Night-Shift Work and Risk of Psoriasis in US Women. Journal of Investigative Dermatology, 133 (2), 565-567. doi: 10.1038/ jid.2012.285

\title{
QUALITY OF LIFE OF PATIENTS WITH OVARIAN CANCER OF THE THIRD STAGE USING DIFFERENTIATED TREATMENT
}

\author{
Andriy Rybin \\ Department of Oncology \\ Odessa National Medical University \\ 2 Valihovsky lane, Odessa, Ukraine, 65082 \\ Andriy.Rybin@gmail.com
}

\begin{abstract}
The aim of this research was to assess the quality of life in patients with stage III ovarian cancer during the treatment period, depending on the applied therapeutic approaches.

A comparative analysis of the results of treatment of 350 patients with adenocarcinoma of the ovaries of III-IV stage, which have undergone suboptimal or non-optimal cytomelective surgery, was conducted. To assess the quality of life and to examine the relationship between changes in physical health and quality of life, patients in both groups were asked to complete the questionnaires EORTC QLQ-C30 and SF-36. According to the questionnaire of the EORTC QLQ-C30, the indicators of physical activity, the ability to do everyday affairs, were higher in the second group of patients who received differentiated treatment. The indicator of "social functioning" was also higher in the II group of patients. When evaluating the patients' liver on the scale of the questionnaire SF-36, it was found that the positive effect of differentiated therapy is maintained for 3 years. Life quality is an important criterion for evaluating the results of antitumor therapy, and when it is continuously monitored in patients with ovarian cancer the safety of treatment for patients could be improved, including at an advanced stage.
\end{abstract}

Keywords: ovarian cancer, treatment, prognostication, quality of life.

\section{Introduction}

Over the past twenty years, the world's scientific community has made a significant progress in the study of quality of life in patients with cancer. The increasing interest in this problem is due to revolutionary changes in the field of medical diagnostic technologies. The introduction of effective cytotoxication methods, the emergence of new chemotherapeutic agents, the widespread use of pharmacogenetic and pharmacogenomic approaches require a clinician to understand the basic laws of sanogenesis and the overall adaptation of the body at various stages of treatment of malignant neoplasms. The National Cancer Institute (NCI) recommends evaluating the effectiveness of treatment of oncopathology according to survival criteria and quality of life (QL) [1]. Evaluating quality of life not only helps in choosing a clinical decision but also avoids unwanted side effects. Data on QL in the dynamics after any medical intervention can improve the interaction of the patient and the physician, identify clinical priorities, including during the rehabilitation and palliative care.

The peculiarities of ovarian cancer (OC) evaluation in patients with ovarian cancer are due to several factors [2]. First of all, OC is an aggressive disease with a low survival rate and a high rate of relapse [3]. This disease is ranked first in terms of mortality among genital cancer in women with OC $46 \%$ for all stages and $31 \%$ for advanced stages [4]. Treatment of OC is based on radical surgical intervention and repeated courses of active chemotherapy. Stress, in which a woman lives 